\title{
Managing the risk of venous thromboembolism in transgender adults undergoing hormone therapy
}

This article was published in the following Dove Press journal:

Journal of Blood Medicine

\author{
Zil Goldstein' \\ Musaub Khan ${ }^{2}$ \\ Tamar Reisman' \\ Joshua D Safer' \\ 'Center for Transgender Medicine and \\ Surgery at Mount Sinai, Mount Sinai \\ Health System and Icahn School of \\ Medicine at Mount Sinai, New York, NY \\ 10029, USA; ${ }^{2} \mathrm{New}$ York Medical College, \\ Valhalla, NY USA
}

\begin{abstract}
Introduction: Venous thromboembolism (VTE) is a potential risk of estrogen therapy. However, data show an improvement in the quality of life for transgender people who use feminizing hormone therapy. With few transgender-specific data, guidance may be drawn from cisgender (nontransgender) data, with a focus on hormonal birth control and postmenopausal hormone replacement therapy (HRT). The aim of this review is to examine the degree to which routes of administration, patient comorbidities, and type of hormone utilized affect the safety of estrogen therapy.
\end{abstract}

Methods: We identified 6,349 studies by searching PubMed with the terms "transgender", "estrogen", "VTE", and "HRT". Of these, there were only 13 studies between 1989 and 2018 that investigated the effects of hormone therapy, including types of estrogens used, in transgender women and men.

Results: The data suggest that the route of hormone administration, patient demographics, and patient comorbidities all affect estrogen's link with VTE. For example, avoiding ethinyl estradiol might make the use of hormone therapy in trans feminine individuals safer than oral birth control. Data from both cis and trans groups suggest additional VTE risk associated with the use of progestins. While transdermal estrogens dosed up to $0.1 \mathrm{mg}$ /day or below appear lower risk for VTE than other forms of estrogen, it is unclear whether this is related to the delivery method or a dose effect. Finally, even if the risk from exogenous estrogen use remains significant statistically, the absolute clinical risk remains low.

Conclusion: Clinicians should avoid the use of ethinyl estradiol. Additionally, data suggest that progestins should be avoided for transgender individuals. Further study of the relationship between estrogen use and the risk of VTE will serve to inform the safest care strategies for transgender individuals.

Keywords: transgender, estrogen therapy, venous thromboembolism

\section{Introduction}

Venous thromboembolism (VTE) is a known risk of hormone therapy. This risk has been well established in the gynecologic literature, specifically in studies of combined oral contraceptives (COCs) and postmenopausal hormone regimens in the cisgender woman. ${ }^{1}$ However, the nuances of VTE risk in transgender individuals using hormone therapy are poorly understood.

Many transgender people use hormone therapy to change their bodies to be more congruent with their gender identity. Data consistently show a significant improvement in the quality of life for transgender people who initiate hormone therapy, which is essential in a population that shows incredibly high rates of attempted suicide. ${ }^{2,3}$ For example, transgender people utilizing hormones reported
Correspondence: Joshua D Safer Mount Sinai Center for Transgender Medicine and Surgery, Icahn School of Medicine at Mount Sinai, CAM Room D240, 17 E. $102^{\text {nd }}$ Street, New York, NY 10029, USA

Tel +I 2126041790

Email jsafer0II5@gmail.com 
lower rates of anxiety relative to those not using hormones, and rates decreased enough to be comparable to the general population. ${ }^{4}$ For trans masculine individuals, those whose sex was recorded female at birth and who identify as men, this involves administration of exogenous testosterone, and data available to date show no increased risk of VTE among these individuals. ${ }^{5}$ Therefore, this review will focus largely on trans feminine individuals, those whose sex was recorded male at birth and who identify as women, where the usual hormone regimens include combinations of estrogens and antiandrogen therapies. $^{6,7}$ Many trans feminine people choose to use feminizing hormone therapy despite possible increased VTE risks. Understanding the risks of hormone therapy for transgender people is essential under these circumstances so clinicians can best guide their patients in identifying and minimizing VTE risks while also improving the quality of trans feminine individuals' lives.

Complicating the interpretation of the available data are considerations regarding the degree that age, estrogen route of administration, and patient comorbidities may affect the VTE risk. Many of the studies do not control for these risk factors. ${ }^{5,7-9}$

\section{Methods}

In accordance with the PRISMA guidelines, we searched PubMed using the terms "transgender", "estrogen", "VTE", and "HRT" (hormone replacement therapy) both through the standard search engine and using MeSH search combinations. From these combinations, we initially found 6,349 studies. We excluded studies that related to the effects of hormone therapy in postmenopausal women and women on birth control, as well as studies that did not analyze the relationship between thromboembolic events and hormone replacement therapy in trans feminine and trans masculine individuals. As a result, the inclusion criteria included studies that mentioned the type of hormone utilized, the route of administration, and patient comorbidities to more comprehensively understand the risk of VTE. We discovered 13 studies between 1989 and 2018 that investigated the effects of hormone therapy, including thrombotic events, in transgender women and men.

\section{Hormone therapy for cisgender people: venous thromboembolism and estrogen}

With few data directly examining the effects of hormone therapy on transgender people, some guidance may be drawn from the available data in cisgender (nontransgender) individuals which focuses on hormonal birth control and postmenopausal hormone replacement therapy.

\section{Hormonal contraceptives}

Most hormonal contraceptives fall into a category termed "combined oral contraceptives" (COCs) which contain a combination of ethinyl estradiol and a progestin. Colloquially, this medication is referred to as "the pill". COCs vary in estrogen dose and progestin formulation. Progestins, in particular, can vary in their estrogenic, antiestrogenic, progestational, antiandrogenic, and androgenic properties. The first documented cases of VTE were published in the 1960s. ${ }^{10}$ Over subsequent years, the link between hormonal contraceptives and VTE has been solidified.

The risk for embolic events in hormonal contraceptive users has been estimated at 3-9 per 10,000 woman-years vs 1-5 per 10,000 woman-years in nonusers. ${ }^{11}$ The use of oral contraceptive drugs increases the risk of VTE 3.8-fold (95\% CI 2.4-6.0). ${ }^{12}$ However, the increased risk of VTE attributable to hormonal contraceptives varies depending on the estradiol dose and progestin type. The highest risk period for VTE occurs with new COC use and in women starting or restarting COC after prolonged discontinuation. ${ }^{13}$ Progestinonly pills are not associated with increased VTE risk. ${ }^{14}$

The initial COCs in the 1950s contained a relatively high ethinyl estradiol content with $50 \mu \mathrm{g}$ per pill. Newer pills contain less ethinyl estradiol, but still may be associated with a higher risk of VTE. ${ }^{15}$ Pills containing the third-generation progestagen desogestrel have a relative risk of VTE of 8.7 compared to 2.2 and 3.8 for COCs with other types of progestogens, such as levonorgestrel. ${ }^{16}$ COCs with $30 \mu \mathrm{g}$ of ethinyl estradiol and desogestrel have an age-adjusted relative risk of 2.5 (95\% CI 1.2-5.2) compared to other COCs, like levonorgestrel. ${ }^{17}$

In particular, the elevated VTE risk associated with drospirenone-containing COCs has been the subject of much debate. Drospirenone is a progestogen that is similar in structure to spironolactone with antimineralocorticoid and antiandrogen effects. Two large European studies demonstrated that, when compared to women taking COCs with levonorgestrel, women taking drospirenonecontaining COCs suffered a significantly higher rate of VTE. ${ }^{18}$ This finding was thought to be related to the observation that aldosterone modifies hemostasis and leads to decreased coagulability. ${ }^{19}$ In light of the apparent connection between drospirenone and VTE, the US Food and Drug 
Administration (FDA) released a statement indicating that COCs containing drospirenone may be associated with a higher risk of VTE compared to other COCs, although the FDA did not establish causality. ${ }^{20}$ A subsequent FDAfunded study published in 2011 came to similar conclusions as the European trials. ${ }^{13}$ By contrast, three large prospective cohort studies, including the European Active Surveillance Study, a US study of 67,000 new COC users, and the International Active Surveillance Study of Women Taking Oral Contraceptives, showed no difference in VTE risk in drospirinone-containing COCs compared to other COCs. ${ }^{21}$ Moreover, women who took 24-day regimens of drospirenone-containing COCs had lower pregnancy rates with typical use than users of 21-day regimens of other progestin-containing COCs. ${ }^{22}$

To put the above findings into perspective, it is important to note that despite the increased risk of VTE in women treated with COCs compared to nonusers, the VTE risk associated with COCs is minimal compared to the risk associated with pregnancy and the postpartum period. Pregnancy is associated with a VTE risk of 5-20/10,000 woman-years, and the postpartum period with a risk of $40-65 / 10,000$ woman-years. ${ }^{13}$ Physiologic changes occurring during pregnancy include an increase in coagulation factors and fibrinogen, as well as a decrease in anticoagulants. ${ }^{23,24}$

The decision to start a patient on hormonal contraception must take into account that individual's personal risk factors for VTE. In particular, much work has been done on characterizing the risks associated with the factor $\mathrm{V}$ Leiden mutation. Compared to women not using the oral contraceptive pill and without factor $\mathrm{V}$ leiden mutation, the risk of women with factor $\mathrm{V}$ leiden mutation who also took the pill was 29-fold higher. ${ }^{12}$

Additional high-risk factors for VTE in COC users include the following:

- Smoking and age $>35$ years

- Less than 21 days postpartum or $21-42$ days postpartum with other risk factors

- Major surgery with prolonged immobilization

- History of deep vein thrombosis or pulmonary embolism

- Hereditary thrombophilia (including antiphospholipid syndrome)

- Inflammatory bowel disease with active or extensive disease, surgery, immobilization, corticosteroid use, vitamin deficiencies, or fluid depletion

- Systemic lupus erythematosus with positive (or unknown) antiphospholipid antibodies
In the above clinical scenarios, the use of hormonal contraception is contraindicated. For other women, the risk of VTE must be balanced with the benefits provided by hormonal contraception such as treatment of menstrual disorders and protection against unwanted pregnancy.

\section{Menopausal hormone replacement and VTE}

Postmenopausal vasomotor symptoms were the first indication for treatment with exogenous estrogen. In 1942, the FDA approved the first exogenous estrogen product, Premarin (a conjugated equine estrogen (CEE)), isolated from pregnant horse urine for the treatment of "hot flashes" associated with menopause.

The Women's Health Initiative was a large observational study conducted by the National Institutes of Health in the 1990s and early 2000s to address health outcomes associated with postmenopausal HRT. Over its course, the study enrolled more than 160,000 women and had a budget of $\$ 625$ million. ${ }^{25}$ Postmenopausal HRT users were found to have 34 clotting events annually per 10,000 women vs nonusers who had a risk of 16 events per 10,000 women. This risk persisted throughout the study and was less apparent in women using estrogen only (meaning they had undergone hysterectomy and thus did not require treatment with progestin to prevent endometrial cancer). Ultimately, the estrogen/progesterone arm of the Women's Health Initiative study was terminated early in 2002 due to concerns of adverse effects. ${ }^{26-28}$

The Women's Health Initiative used oral conjugated estrogens (Premarin) and medroxyprogesterone acetate in its estrogen/progesterone arm, which is of specific interest when discussing VTE risk. It is now thought that the method of estrogen administration impacts VTE outcomes. The ESTHER (Estrogen and Thromboembolism Risk) study suggested that oral, but not transdermal, estradiol increases the risk of VTE in postmenopausal women. ${ }^{29}$ Oral estrogens have a significant first-pass effect, which is possibly what causes their disproportionate thrombogenic effects. Oral estrogen increases levels of factor VII, factor VIII, factor X, prothrombin, and fibrinogen, and decreases antithrombin and protein S. $^{30-32}$ However, available data compare nonequivalent doses of transdermal and oral estrogens, so it is unclear whether this is dose related or route of administration related.

\section{Transgender women and HRT}

Analysis of the noted studies suggests that the type of oral estrogen, hormone route of administration, and patient 
demographics and comorbidities may affect estrogen's link with VTE (Table 1). The data also suggest that myocardial infarction is less frequently observed than stroke, and venous disease is most prevalent. $5,33,34$

Different types of estrogen may be associated with different risk profiles. Ethinyl estradiol, the most common estrogen in COC pills, has the most data suggesting a link to VTE. A long-term retrospective study following hormone treatments in 966 transgender women for a median of 18.5 years found that oral ethinyl estradiol is independently associated with a 3-fold increase in cardiovascular death, including by VTE. ${ }^{9}$ A cohort study of 816 transgender women further found venous thromboembolic incidents in 41 patients $(5.5 \%)$, with 40 of those individuals taking oral ethinyl estradiol and one prescribed transdermal estrogen with a prior history of VTE. ${ }^{8}$

By contrast, CEEs and 17 $\beta$-estradiol appear much safer. A cohort of 61 transgender women who were prescribed CEEs did not suffer any venous thromboembolic events, and neither did another group of 23 transgender women who were taking an oral estradiol regimen. ${ }^{34}$ In one study, however, it was shown that CEEs were correlated with a higher VTE risk than estrogen valerate or ethinyl estradiol. ${ }^{35}$ While these data suggest the relative safety of various oral estrogens relative to ethinyl estradiol, more complete trials need to be conducted to further understand their safety. However, it seems the case that different oral estrogens do offer varying risk for transgender women, and this is important to consider when evaluating the cardiovascular risk of hormone therapy.

Transdermal estrogen, whether patch or gel, has been reported to have the least thrombogenic profile in transgender women, although there are no head-to-head studies with other estrogen products. In a study of 162 transgender women prescribed transdermal estrogen for an average of 4.4 years, no VTE occurred. ${ }^{36}$ This is particularly significant as 18 study participants were identified to have thrombophilic genetic mutations at the initiation of hormone therapy.

Oral estrogens seem to have similar effects on surrogate endpoints in transgender women as they do in cisgender women. In 23 transgender women taking Premarin over a span of 6 months, proinflammatory cytokines IL-1, IL-6, IL8 , and tumor necrosis factor- $\alpha$ were elevated. ${ }^{37}$ Transdermal estrogen, on the other hand, has not been reported to increase proinflammatory cytokines and procoagulant factors, and this is further corroborated in transgender women. ${ }^{37-39}$

The pharmacokinetics of transdermal and oral estrogen could support a model where transdermal estrogens are safer. However, studies to date do not consider serum estradiol levels or doses of estrogens used as a potential contributing risk factor for VTE. All available data use nonequivalent doses of oral and transdermal estradiol when making these comparisons. Clinicians often use doses up to $0.2-0.3 \mathrm{mg} /$ day of transdermal estradiol, and the highest dose studied is $0.1 \mathrm{mg} /$ day.

Patient demographics and comorbidities also affect their venous thromboembolic risk profile, including age, smoking habits, hypertension, thrombophilic conditions, history of thromboses, and mental illness, among others.

Many of the studies analyzed report coexisting conditions that increase the study participants' risk for VTE. Variables that have been noted to exacerbate VTE risk include smoking, HIV, malignancies, high cholesterol, clotting disorders, and hypertension. One study showed that all 11 patients who suffered thromboembolic phenomena were smokers, underwent recent surgery, and/or suffered from dyslipidemia or hypertension. ${ }^{5}$ Only one study to date demonstrates an occurrence of VTE in the absence of risk factors beyond hormone therapy. ${ }^{40}$

The duration of hormone use has been associated with an increased risk of VTE. The largest retrospective study to date found that among the 61 cases (2\% of the trans feminine people on estrogen therapy in the cohort) who experienced VTE, there was a 4-fold risk increase in the women who had been taking estrogen for 8 years compared to those who had been taking estrogen for 2 years. ${ }^{33}$ This study controlled for smoking status, blood pressure, and history of cardiovascular events but did not address HIV, which could be a possible confounding variable between these groups. Additionally, a significant majority of the participants did not have the dose or method of administration recorded for their estrogen therapy.

Data suggest a positive correlation between estrogen use and VTE. A recent review found the overall incidence rate to be 2.3 events per 1,000 patient-years. ${ }^{41}$ While this is a significant increase in risk, the absolute risk remains relatively low and often does not dissuade patients from initiating feminizing hormone therapy.

\section{Conclusion}

While data on VTE risk in trans feminine individuals on hormone therapy are underdeveloped, there are several important clinical lessons to be learned. Clinicians should avoid the use of ethinyl estradiol as it has a significantly higher risk for VTE than other formulations of estrogen. Avoiding ethinyl estradiol might make the use of hormone therapy in trans 


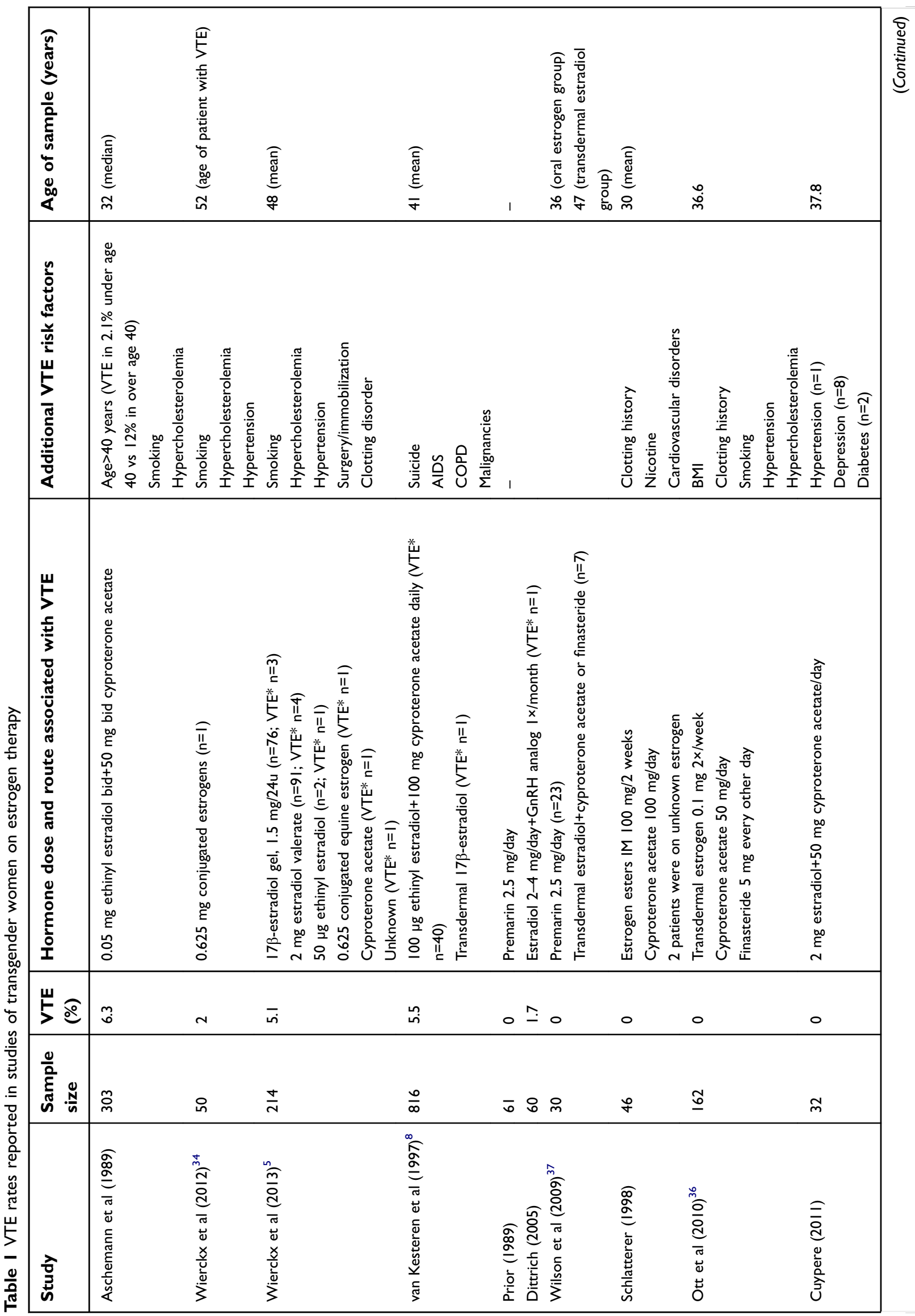


feminine individuals safer than birth control. Additionally, data in both cis and trans groups suggest an additional VTE risk associated with the use of progestins, which would suggest they be avoided when working with transgender individuals. Ethinyl estradiol has largely been abandoned as a form of hormone therapy; however, current data do not offer more specific insight into how to quantify cardiovascular risk. Although there seems to be clear evidence that transdermal estrogens dosed up to $0.1 \mathrm{mg} /$ day or below are a lower risk for VTE than other forms of estrogen, it is unclear whether this is related to the delivery method or a dose effect. Risk mitigation is an important part of the care of transgender patients due to the many risks associated with not providing hormone therapy (ie, poor mental health) and the potential risks associated with hormone therapy. Further study of the relationship between estrogen and the risk of VTE will serve to inform the safest possible care for transgender patients. ${ }^{42}$

\section{Disclosure}

Dr Joshua D Safer was a member of an Advisory Board for Endo Pharmaceuticals, June, 2018. The authors report no other conflicts of interest in this work.

\section{References}

1. Gomes MPV, Deitcher SR. Risk of venous thromboembolic disease associated with hormonal contraceptives and hormone replacement therapy: a clinical review. Arch Intern Med. 2004;164(18):1965. doi:10.1001/archinte.164.18.1965

2. Hess J, Breidenstein A, Henkel A, et al. Satisfaction, quality of life and psychosocial resources of male to female transgender after gender reassignment surgery. Eur Urol Suppl. 2018;17(2):e1748. doi:10.1016/ S1569-9056(18)32062-1

3. S. E. James, et al. The report of the 2015 U.S. Transgenderr Survey. National Center for Transgnder Equality; 2016. Available from: https://transequality.org/sites/default/files/docs/usts/USTS-Full-

Report-Dec17.pdf. Accessed June 10, 2019.

4. Heylens, Gunter, Verroken C, De Cock S, T'Sjoen G, De Cuypere G. Effects of different steps in gender reassignment therapy on psychopathology: a prospective study of persons with a gender identity disorder. J Sex Med. 2014;11(1):119-126.

5. Wierckx K, Elaut E, Declercq E, et al. Prevalence of cardiovascular disease and cancer during cross-sex hormone therapy in a large cohort of trans persons: a case-control study. Eur J Endocrinol. 2013;169(no. 4):471-478. doi:10.1530/EJE-13-0493

6. Asscheman H, Giltay EJ, Megens JAJ, de Ronde W, van Trotsenburg MAA, Gooren LJG. A long-term follow-up study of mortality in transsexuals receiving treatment with cross-sex hormones. Eur J Endocrinol. 2011;164(4):635-642. doi:10.1530/EJE$10-1038$

7. Getahun D, Nash R, Flanders WD, et al. Cross-sex hormones and acute cardiovascular events in transgender persons: a cohort study. Ann Intern Med. 2018;169:205. doi:10.7326/M17-2785

8. van Kesteren PJM, Asscheman H, Megens JAJ, Gooren LJG. Mortality and morbidity in transsexual subjects treated with crosssex hormones. Clin Endocrinol (Oxf). 1997;47(3):337-343. doi:10.1046/j.1365-2265.1997.2601068.x 
9. Asscheman, Asscheman H, Giltay EJ, et al. A long-term follow-up study of mortality in transsexuals receiving treatment with cross-sex hormones. Eur J Endocrinol. 2011;164:635-642. doi:10.1530/EJE-10-1038

10. Tyler ET. Oral contraception and venous thrombosis. JAMA. 1963;185(2):131. doi:10.1001/jama.1963.03060020091034

11. American College of Obstetricians and Gynecologists. Risk of venous thromboembolism among users of drospirenone-containing oral contraceptive pills. Committee Opinion No. 540. Obstetrics Gynecol. 2012;120:1239-1242.

12. Vandenbroucke JP, Koster T, Rosendaal FR, Briët E, Reitsma PH, Bertina RM. Increased risk of venous thrombosis in oral-contraceptive users who are carriers of factor V Leiden mutation. Lancet. 1994;344(8935):1453-1457. doi:10.1016/S0140-6736(94)90286-0

13. Sidney S, Cheetham TC, Connell FA, et al. Recent combined hormonal contraceptives (CHCs) and the risk of thromboembolism and other cardiovascular events in new users. Contraception. 2013;87 (1):93-100. doi:10.1016/j.contraception.2012.09.015

14. Le Moigne E, Tromeur C, Delluc A, et al. Risk of recurrent venous thromboembolism on progestin-only contraception: a cohort study. Haematologica. 2016;101(1):e12-e14. doi:10.3324/haematol.2015. 134882

15. FDA drug safety communication: updated information about the risk of blood clots in women taking birth control pills containing drospirenone. UCM (US Food and Drug Administration). Available from: https://www.fda.gov/Drugs/DrugSafety/ucm299305. Accessed December 3, 2018.

16. Vandenbroucke JP, Helmerhorst FM, Bloemenkamp KWM, Rosendaal FR. Third-generation oral contraceptive and deep venous thrombosis: from epidemiologic controversy to new insight in coagulation. Am J Obstet Gynecol. 1997;177(4):887-891. doi:10.1016/ S0002-9378(97)70289-8

17. Vandenbroucke JP, Koster T, Briët E, Reitsma PH, Bertina RM, Rosendaal FR. Increased risk of venous thrombosis in oralcontraceptive users who are carriers of factor V Leiden mutation. Lancet. 1994;344(8935):1453-1457

18. O. Lidegaard, et al. Hormonal contraception and risk of venous thromboembolism: national Follow-up Study. Bmj. 2009;339(aug 13 2):b2890-b2890. doi:10.1136/bmj.b2890

19. Ducros E, Berthaut A, Mirshahi SS, et al. Aldosterone modifies hemostasis via upregulation of the protein-c receptor in human vascular endothelium. Biochem Biophys Res Commun. 2008;373(2):192196. doi:10.1016/j.bbrc.2008.05.185

20. FDA drug safety communication: updated information about the risk of blood clots in women taking birth control pills containing drospirenone. UCM (US Food and Drug Administration). Available from: https://www. fda.gov/Drugs/DrugSafety/ucm299305. Accessed December 3, 2018.

21. Seeger JD, Loughlin J, Eng PM, Clifford CR, Cutone J, Walker AM. Risk of thromboembolism in women taking Ethinylestradiol/ Drospirenone and other oral contraceptives. Obstetrics Gynecol. 2007;110(3):587-593. doi:10.1097/01.AOG.0000279448.62221.a8

22. Dinger J, Do Minh T, Buttmann N, Bardenheuer K. Effectiveness of oral contraceptive pills in a large US cohort comparing progestogen and regimen. Obstetrics Gynecol. 2011;117(1):33-40. doi:10.1097/ AOG.0b013e31820095a2

23. World Health Organization. Combined hormonal contraceptive use during the postpartum period. January 26, 2010. Available from: https://apps who.int/iris/bitstream/handle/10665/70447/WHO_RHR_10.15_eng.pdf; jsessionid=A2053BAFEA3D15DF51D82559E9973599? sequence=1. Accessed June 10, 2019.

24. Jackson E, Curtis KM, Gaffield ME. Risk of venous thromboembolism during the postpartum period: a systematic review. Obstetrics Gynecol. 2011;117:691-703. doi:10.1097/AOG.0b013e31820ce2db
25. Parker-Pope T, The women's health initiative and the body politic. The New York Times. 2011 Apr 9.

26. Burwen DR, Wu C, Cirillo D, et al. Venous thromboembolism incidence, recurrence, and mortality based on women's health initiative data and medicare claims. Thromb Res. 2017;150:78-85. doi:10.1016/j.thromres.2016.11.015

27. Canonico M, Plu-Bureau G, O’Sullivan MJ, et al. Age at menopause, reproductive history, and venous thromboembolism risk among postmenopausal women: the women's health initiative hormone therapy clinical trials. Menopause. 2014;21(3):214-220. doi:10.1097/ GME.0b013e31829752e0

28. Curb JD, Ross LP, Bray PF, et al. Venous thrombosis and conjugated equine estrogen in women without a uterus. Arch Intern Med. 2006;166(7):772-780. doi:10.1001/archinte.166.7.772

29. Canonico M, Oger E, Plu-Bureau G, et al. Hormone therapy and venous thromboembolism among postmenopausal women: impact of the route of estrogen administration and progestogens: the ESTHER study. Circulation. 2007;115(7):840-845. doi:10.1161/ CIRCULATIONAHA.106.642280

30. Conard J. Biological coagulation findings in third-generation oral contraceptives. Hum Reprod Update. 1999;5(6):672-680. doi:10.1093/humupd/5.6.672

31. Oger E, Alhenc-Gelas M, Lacut K, et al. Differential effects of oral and transdermal Estrogen/Progesterone regimens on sensitivity to activated Protein C among postmenopausal women: a randomized trial. Arterioscler Thromb Vasc Biol. 2003;23(9):1671-1676. doi:10.1161/01.ATV.0000087141.05044.1F

32. Deguchi H, Bouma BN, Middeldorp S, Lee YM, Griffin JH. Decreased plasma sensitivity to activated protein $\mathrm{c}$ by oral contraceptives is associated with decreases in plasma glucosylceramide. JTH. 2005;3(5):935-938. doi:10.1111/j.1538-7836.2005.01335.x

33. Getahun D, Nash R, Flanders WD, et al. Cross-sex hormones and acute cardiovascular events in transgender persons: a cohort study. Ann Intern Med. 2018;169:205-213. doi:10.7326/M17-2785

34. Wierckx K, Mueller S, Weyers S, et al. Long-term evaluation of cross-sex hormone treatment in transsexual persons. J Sex Med. 2012;9(10):2641-2651. doi:10.1111/j.1743-6109.2012.02876.x

35. Seal LJ, Franklin S, Richards C, Shishkareva A, Sinclaire C, Barrett J. Predictive markers for mammoplasty and a comparison of side effect profiles in transwomen taking various hormonal regimens. J Clin Endocrinol Metab. 2012;97(12):4422-4428. doi:10.1210/jc.2012-2030

36. Ott J, Kaufmann U, Bentz E-K, Huber JC, Tempfer CB. Incidence of thrombophilia and venous thrombosis in transsexuals under cross-sex hormone therapy. Fertil Steril. 2010;93(4):1267-1272. doi:10.1016/j. fertnstert.2008.12.017

37. Wilson R, Spiers A, Ewan J, Johnson P, Jenkins C, Carr S. Effects of high dose oestrogen therapy on circulating inflammatory markers. Maturitas. 2009;62(3):281-286. doi:10.1016/j. maturitas.2009.01.009

38. Lowe GDO. Hormone replacement therapy and cardiovascular disease: increased risks of venous thromboembolism and stroke, and no protection from coronary heart disease. J Intern Med. 2004;256 (5):361-374. doi:10.1111/j.1365-2796.2004.01400.x

39. Vongpatanasin W, Tuncel M, Wang Z, Arbique D, Mehrad B, Jialal I. Differential Effects of Oral versus Transdermal Estrogen Replacement Therapy on C-Reactive Protein in Postmenopausal Women. J Am Coll Cardiol. 2003;41(8):1358-1363. doi:10.1016/ S0735-1097(03)00156-6

40. Arnold JD, Sarkodie EP, Coleman ME, Goldstein DA. Incidence of venous thromboembolism in transgender women receiving oral estradiol. J Sex Med. 2016;13(11):1773-1777. doi:10.1016/j. jsxm.2016.09.001 
41. Khan J, Schmidt RL, Spittal MJ, Goldstein Z, Smock KJ, Greene DN. Venous thrombotic risk in transgender women undergoing estrogen therapy: a systematic review and meta-analysis. Clin Chem. 2019;65(1):57-66.
42. Defreyne J, Van de Bruaene LDL, Rietzschel E, Van Schuylenbergh J, T'Sjoen GGR. Effects of gender-affirming hormones on lipid, metabolic, and cardiac surrogate blood markers in transgender persons. Clin Chem. 2019;65(1):119-134. doi:10.1373/clinchem.2018.288241

\section{Publish your work in this journal}

The Journal of Blood Medicine is an international, peer-reviewed, open access, online journal publishing laboratory, experimental and clinical aspects of all aspect pertaining to blood based medicine including but not limited to: Transfusion Medicine; Blood collection, Donor issues, Transmittable diseases, and Blood banking logistics; Immunohematology; Artificial and alternative blood based therapeutics; Hematology; Biotechnology/nanotechnology of blood related medicine; Legal aspects of blood medicine; Historical perspectives. The manuscript management system is completely online and includes a very quick and fair peer-review system. Visit http://www.dovepress.com/testimonials.php to read real quotes from published authors. 